\title{
Tissue Fragility and Ehlers-Danlos Syndrome Global Aggravation by Osteopathic Manipulations
}

\author{
Claude Hamonet ${ }^{1,2 *}$, Geoffroy Nourrissat ${ }^{3}$, David Cypel ${ }^{4}$ and Lucette Ducret ${ }^{4}$ \\ ${ }^{1}$ Université Paris-Est-Créteil, France \\ ${ }^{2}$ Centre de santé ELLAsanté, Paris
}

${ }^{3}$ Clinique Maussins-Nollet, Paris

${ }^{4}$ Saint-Paul-en Chablais, France

*Corresponding author: Claude Hamonet, Université Paris-Est-Créteil, France

\begin{abstract}
Ehlers-Danlos syndrome is a hereditary, diffuse connective tissue disorder whose frequency is largely underestimated. Its diagnosis rests on a grouping of clinical criteria while biogenetic results are unreliable. Paradoxically, Ehlers-Danlos syndrome is considered as a rare disease while its prevalence in the world is at least $2 \%$. It is very poorly known and very rarely diagnosed. This situation is dangerous for patients and exposes them to iatrogenic effects. The case reported here is a good illustration of this situation in a 26-year-old man, with local complications (paralysis of serratus major), a dramatic worsening of the disease's symptoms (pain, proprioception) and exclusion from the work world.
\end{abstract}

Keywords: Ehlers-Danlos; Joint Hypermobility; Rachis Manipulation; Serratus Major Palsy; Charles Bell Nerve; Osteopathy; Pain; Proprioception

\section{Introduction}

Ehlers-Danlos disease, despite its frequency, remains largely unheard of and is almost never diagnosed. Thus, Professor Rodney Grahame [1] of the London University says: "It is incomprehensible that most physicians, specialists or not, do not track it, do not know how to diagnose it or, of course, how to treat it. Many, even, deny its existence, often leading the patient and his family into dramatic and unnecessary suffering". It is characterized by a hereditary modification of the structure of all connective tissues including bone and vascular systems. The nervous system is unhurt, but it is confronted with a body that sends inappropriate signals for which it is not programmed. Indeed, sensors are placed in pathological tissues which disrupt their biomechanical responses to solicitations. Clinical manifestations of Ehlers-Danlos syndrome then appear as the consequences of proprioception disorder [2,3]. Joint disorders, inter alia, are one of the clinical expressions that lead many of these patients to resort to osteopathy. The second consequence of Ehlers-Danlos syndrome's collagenous tissues particularities is their fragility. Skin is thin and heals badly, small vessels break easily and can cause difficult to control hemorrhages. Excessive laxity and loss of elasticity have consequences for shock waves that are easily transmitted without being damped, which is often observed after major trauma, especially in traffic accidents. Shock waves can sometimes produce brain changes that can be objectified by diffusion tensor MRI [4]. It is the same, after vertebral manipulation, especially cervical. We report here an observation of significant worsening of the clinical picture, combining proprioceptive disorders and pain, as well as a paralysis of serratus major nerve, after spine manipulation in a man suffering from Ehlers-Danlos syndrome. Disease diagnosis was made when disabling functional symptoms appeared, triggered by manipulation.

\section{Clinical History}

This 26-year-old man was considered healthy until January 04, 2019. He practiced boxing, judo, as well as dancing. He mentions 
some ankle sprains, torticollis, frequent muscle cramps and dislocation of the right shoulder, which was surgically treated with a coracoid bony abutment, using Latarget's technique, four years earlier. In December 2016, he consulted an osteopath for neck and back pain with movement limitation. Four osteopathic maneuvers are performed. The first maneuver, in sitting position: osteopath placed behind the patient, raises the patient's arms, with both hands flat on the skull's posterior face. Then, he exercises, several sudden thrusts until crunching. The second maneuver, in right lateral decubitus, pelvis blocked by the manipulator's knee that pulls on the right upper limb by bringing it upwards and forwards, in association with a rotational movement of the top of the trunk. The third maneuver, the patient is also in left lateral decubitus, pelvis blocked by the osteopath's knee on the patient's lower limbs in flexion, left shoulder blocked by the weight of the patient's body. The manipulator exerts a pressure on the lower part of the lumbar region in order to mobilize it. The fourth maneuver, finally, still on the left side, right thigh flexed to the maximum, is a mobilization of the whole trunk.

\section{Immediately after these Maneuvers, the Patient Complains of the Following Manifestations:}

a) Feeling of right shoulder's instability that "no longer holds", associated with hindrance of its mobilization. This shoulder appears to him as "different".

b) Diffuse headaches associated with tinnitus (whistling) and mental clouding.

c) Pain in the neck and the entire back, making it very difficult for the patient to hold his head.

d) Respiratory discomfort with sensation of difficulty in "opening" the thorax.

e) Difficulties in walking in straight line, in leaning on the left side. He cannot rotate to the left anymore. He feels insecure while walking, which is painful and, in the days that follow is completely unable to walk. He is forced to rent a manual wheelchair to leave his home and keep some social contacts, but he must stop working.

f) Standing position is difficult and cannot exceed a few minutes, sitting position is also painful. He must adopt the position "inclined as on a deckchair" to relieve his back. Only the position lying on the ground, eases his pain during crises. He must go several times to hospital emergencies, without lasting success on his functional difficulties.

This clinical picture dominated by pain has but few treatments except for Tramadol and opiates which provide a very relative comfort on his pain's intensity but does not help with his mobility. On several occasions, the phrase "it's hell" came back into the conversation, expressing the intensity of the endured suffering. The patient had never previously experienced such symptoms. After six months, the patient decides to seek an orthopedic surgeon's advice. The physician prescribes an electromyogram, which shows right serratus major muscle paralysis with reinnervation in progress, explaining the felt instability of the right shoulder blade. Imaging shows disappearance of the coracoid graft which has completely melted [5]. A new surgical procedure is performed with tensioning of the tissues maintaining the scapulo-humeral joint. Ablation of the screws, put in place to fix the graft during the first operation, turned out to be impossible. Surgery is followed by shoulder physiotherapy, which has contributed to the recovery of serratus major paralysis, which is known to be slow due to the length of the injured nerve [6]. At the time of our examination, this muscle has a score close to normal $(4 / 5)$ at muscular testing (resistance, arm extended, at the lowering of the arm). The patient has still not returned to work.

\section{Evidence of Ehlers-Danlos Disease}

In the patient's antecedents and the event's relation which followed the osteopathic manipulations, quite a few elements draw attention: notion of articular hypermobility, existence of a dislocation of the right shoulder, operated, clinical symptoms described after manipulation (diffuse and very intense pain, marked proprioceptive disorders leading to use a wheelchair). These signs suggest a diffuse hereditary connective tissue disease: EhlersDanlos-Tschernogobow syndrome. Diagnosis confirmation is made by finding 5 signs at least out of 9 significant clinical signs with a sensitivity of $99.6 \%$ and a specificity of $97,1 \%$ from a mathematical modeling of the signs performed with a population of 853 patients with Ehlers-Danlos syndrome [7]. The nine signs are: diffuse pain, severe fatigue, thin and fragile skin, proprioceptive motor disorders, joint instability, joint hypermobility, gastroesophageal reflux, frequent bruising and hyperacusis (or vertigo). These nine signs are present in this patient. Other signs related to this pathology are also found such as dystonia explaining contracture crises and involuntary "twitching", occurring during the day and at the time of falling asleep and vasomotor disorders (cold feet, heavy sweating, chills, hypotension, palpitations).

\section{Discussion}

The existence of Ehlers-Danlos disease strictly contraindicates spine manipulation but also peripheral joints manipulation. They can cause serious complications (neck arteries dissection, craniocervical dislocation due to the presence of a Chiari syndrome) and local worsening (pain, joint instability, Charles Bell nerve, ulnar nerve, brachial plexus in the parade of scalene stretch), but also of all the syndrome's symptoms: fatigue, pain, dysproprioception, dystonia, diplopia, pseudo paralysis, dysautonomia. These aggravations can persist over a long period, or even permanently, causing the patient to fall from functional discomfort to loss of functional and social autonomy. This poses medico-legal problems involving the responsibility of the manipulations' author and of the prescriber or adviser.

This Type of Accident also Raises the Problem of Diagnosing a Common but Rarely Diagnosed or Very Late in Time, a Disease Which is Present from Birth. The Reasons are Plural:

a. The multiplicity of symptoms confuses specialists trained in organs' pathology who have many difficulties to consider that combination of fragile skin, hyperacusis and significant 
fatigue is suggestive of the diagnosis. It is precisely because it has many apparently disparate clinical manifestations that we must mention this disease that affects all organs containing collagen.

b. Another reason arising from the first is historical construction [8] of this disease's description. The first descriptors were dermatologists who focused on two signs: excessive skin stretching (cutis laxa) and joints hypermobility. One of them (Danlos) has unfortunately mistakenly described pseudo elastic xanthoma [9] in which the skin is very stretchable, which is not the case for all patients in EhlersDanlos syndrome. This contributes, until today, as we observe it regularly, to eliminate a diagnosis, otherwise obvious, if skin's stretchability is considered insufficient. "You do not have Ehlers-Danlos syndrome," hears the patient who can put his hands flat on the floor without bending his knees and suffers from multiple troubles. In this last maneuver, presence of retractions, especially of the knee's flexor muscles and triceps surrae muscle is frequent and constitutes, in the child, a sign in favor of this diagnosis [10]. In the syndrome descriptions, a very important part was made to joint hypermobility. A geneticist, Beighton [11], proposed an evaluation test, rated on nine points. It is unfortunately limited to 5 articular groups (fingers, elbows, knees, thoracolumbar spine) neglecting the most affected joints (shoulders, hips, neck). Based on this test, Beighton and his successors tried to classify the signs into three [12], then eleven [11], then six [13] and now [14] thirteen different forms of a disease that is one, as clinical observations clearly show. They hypothesized that there was a link between genetic mutations and the groups of observed symptoms. Very often, there is no detectable genetic modification. Other authors have proposed new measurement criteria, including shoulder [15]. It is mainly on joint mobility that the diagnosis is constructed, neglecting many other symptoms including cognitive skills that sometimes lead to dyslexia and autistic behavior [16].

Beyond iatrogenic accidents, two unfortunate consequences are observed: transmission of the disease by parents who do not know the risk and parents' accused of violence because their child presents hemorrhages or spontaneous fractures with very heavy judicial and educational consequences [17]. Finally, making the diagnosis, leads to offer treatments (oxygen therapy, special clothing and orthoses for proprioceptive purpose, anti-dystonic medication, appropriate painkillers, physiotherapy, occupational therapy, speech therapy, adapted physical activities) which have proved effective [18] and have truly "changed the lives" of many of these patients.

\section{Conclusion}

Paradoxically, Ehlers-Danlos syndrome, a common inherited systemic disorder, described over the last 140 years [19], is undiagnosed because its symptomatology is multiple, poorly described and un-taught. Moreover, there is no formal genetic test, the only biological test being electron microscopy which objectifies collagen fibrils abnormalities [20,21]. Among therapeutic actions at risk are spinal manipulations that tend to multiply with current osteopathy development. This disease's joint symptomatology drives patients to turn to this type of therapy. The risks for these patients are very important, as this clinical case shows.

\section{References}

1. Hamonet C Ehlers-Danlos (2018) the disease forgotten by medicine Harmattan 2018 Paris Introduction Grahame R.

2. Holly A Clayton, Stephanie AH Jones, Denise HP Henriques (2015) Proprioceptive precision is impaired in Ehlers-Danlos syndrome. Springerplus 4: 323.

3. Deparcy D (2016) Proprioception and posture in Ehlers-Danlos syndrome. Journal of Medical Rehabilitation: Practice and Training in Physical Medicine and Rehabilitation 36(1): 38-42.

4. Claude Hamonet C, Frédy D, Lefèvre JH, Bourgeois-Gironde S, Zeitoun JD (2016) Brain injury unmasking Ehlers-Danlos syndromes after trauma: the fiber print. Orphanet J Rare Dis 11: 45.

5. Nourissat G, Vigan M, Hamonet C, Doursounian L, Deranlot J (2018) Diagnosis of Ehlers-Danlos syndrome after a first shoulder dislocation. J Shoulder Elbow Surg 27(1): 65-69.

6. Grossiord A, Hamonet Cl, Lacert P, Alexandre JH (1969) Paralysis of the large dentate (about 20 cases). Rev Neurol 121: 605-613.

7. Hamonet C, Brissot R, Gompel A, Guinchat V, Brock I, et al. (2018) EhlersDanlos Syndrome-Contribution to clinical diagnosis- About 853 cases. EC neurology 10.6 (2018): 428-439.

8. Hamonet C, Ducret L, Baeza-Velasco L, Layadi K, Ehlers-Tschernogobow (2016) Annoyed History of the Disease, History of Medical Sciences 50: $29-41$.

9. Danlos A (1908) A case of cutaneous laxa with tumors by chronic contusion of the elbows and knees (pseudo-diabetic juvenile xanthome by MM Hallopeau and Macé de 1 Epinay). Bull soc Fr Dermatol 19: 70-72.

10. Hamonet C, Grossin D (2016) Rétractions musculo-tendineuses et syndrome d'Ehlers-Danlos. Journal de réadaptation médicale 36(3): 168-170.

11. Beighton P, De Paepe A, Danks D, Finidori G, Gedde-Dahl T, et al. (1988) International nosology of heritage disorders of connective tissue, Berlin, 1986. Am J Med Genet 29(3): 581-594.

12. Barrabas AP (1967) Heterogeneity of the Ehlers-Danlos syndrome: Description of the three clinical types and a hypothesis to explain the basic defect(s). Br Med J 2(5552): 612-613.

13. Beighton P, De Paepe A, Steinma B, Tsipouras P, Wenstrup RJ (1998) Ehlers-Danlos revised nosology, Villefranche, 1997. Ehlers-Danlos National Foundation and Ehlers-Danlos support group (UK). American Journal of Medical Genetics 77(1): 31-37.

14. Malfait F, Francomano C, Byers P, Belmont J, Berglund B, et al. (2017) American Journal of Medical Genetics Part C: Seminars in Medical Genetics Supplement to the American Journal of Genetics. 175(1): 1-2.

15. Cypel D (2019) Measurement of glenohumeral abduction in patients with Ehlers-Danlos syndrome. Journal of Orthopedic and Traumatological Surgery 105(2): 163-169.

16. Sieg KG (1992) Autism and Ehlers-Danlos syndrome. J Am Acad Child Adolesc Psychiatry 31(1): 173.

17. Baeza-Velasco C, Cohen D, Claude Hamonet C, Lautaro Diaz L, Cravero C, et al. (2018) Autism, Joint Hypermobility-Related Disorders and Pain. Front Psychiatry 9: 656.

18. Michael F Holick, Tabatabaei F, Hossein-Nezhad A (2017) Multiple fractures in infants who have Ehlers-Danlos/hypermobility syndrome and or vitamin D deficiency: A case series of 72 infants whose parents were accused of child abuse and neglect. Dermato-Endocrinology 9(1): e1279768. 
19. Hamonet C, Manicourt D (2018) How to prevent and treat Ehlers-Danlos syndrome? A medico-social emergency. A twenty years' experience. International Symposium on the Ehlers-Danlos Syndromes. Ghent (Belgium), p. 26-29.

20. Chernogubow NA (1892) About a case of Cutis laxa. (Presentation at the first meeting of Moscow Dermatologic and Venerologic Society) 14: 76.
21. Hamonet C, Manicourt D, Hermanns-Lê T, Pommeret S (2018) Clinical diagnosis of Ehlers-Danlos syndrome, new insights into the clinical setting. International Symposium on the Ehlers-Danlos Syndromes. Ghent (Belgium), p. 26-29.

\section{(C) (i) This work is licensed under Creative}

To Submit Your Article Click Here:

Submit Article

DOI: $10.32474 / 0 J N B D .2019 .03 .000159$

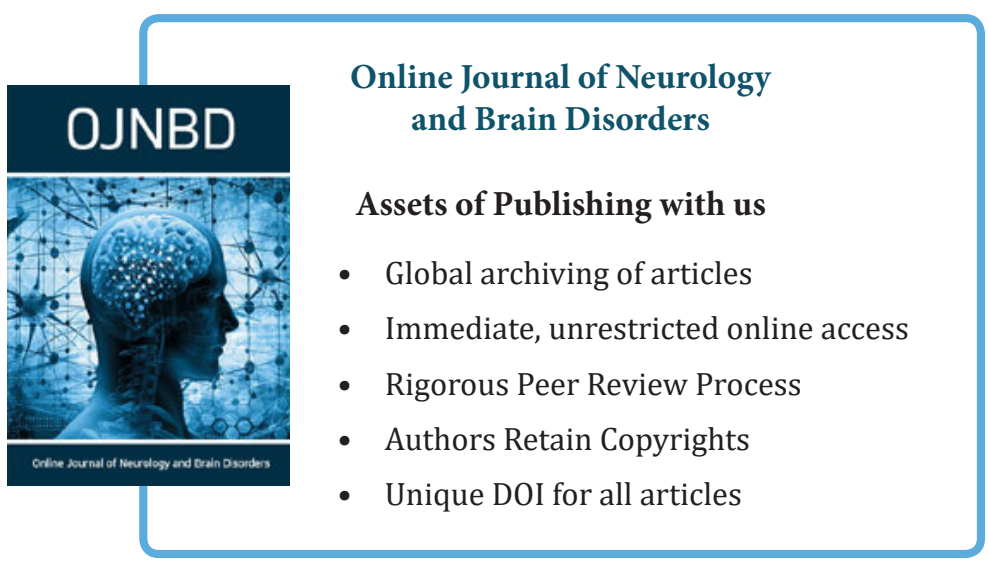

